Document downloaded from:

http://hdl.handle.net/10251/64832

This paper must be cited as:

Montes Robles, R.; Moragues Pons, ME.; Vivancos Bono, JL.; Ibáñez Civera, FJ.; Martínez Mañez, R.; Fraile Muñoz, R.; García Breijo, E. (2015). Colorimetric detection of hazardous gases using a remotely operated capturing and processing system. ISA Transactions. 59:434-442. doi:10.1016/j.isatra.2015.09.010.

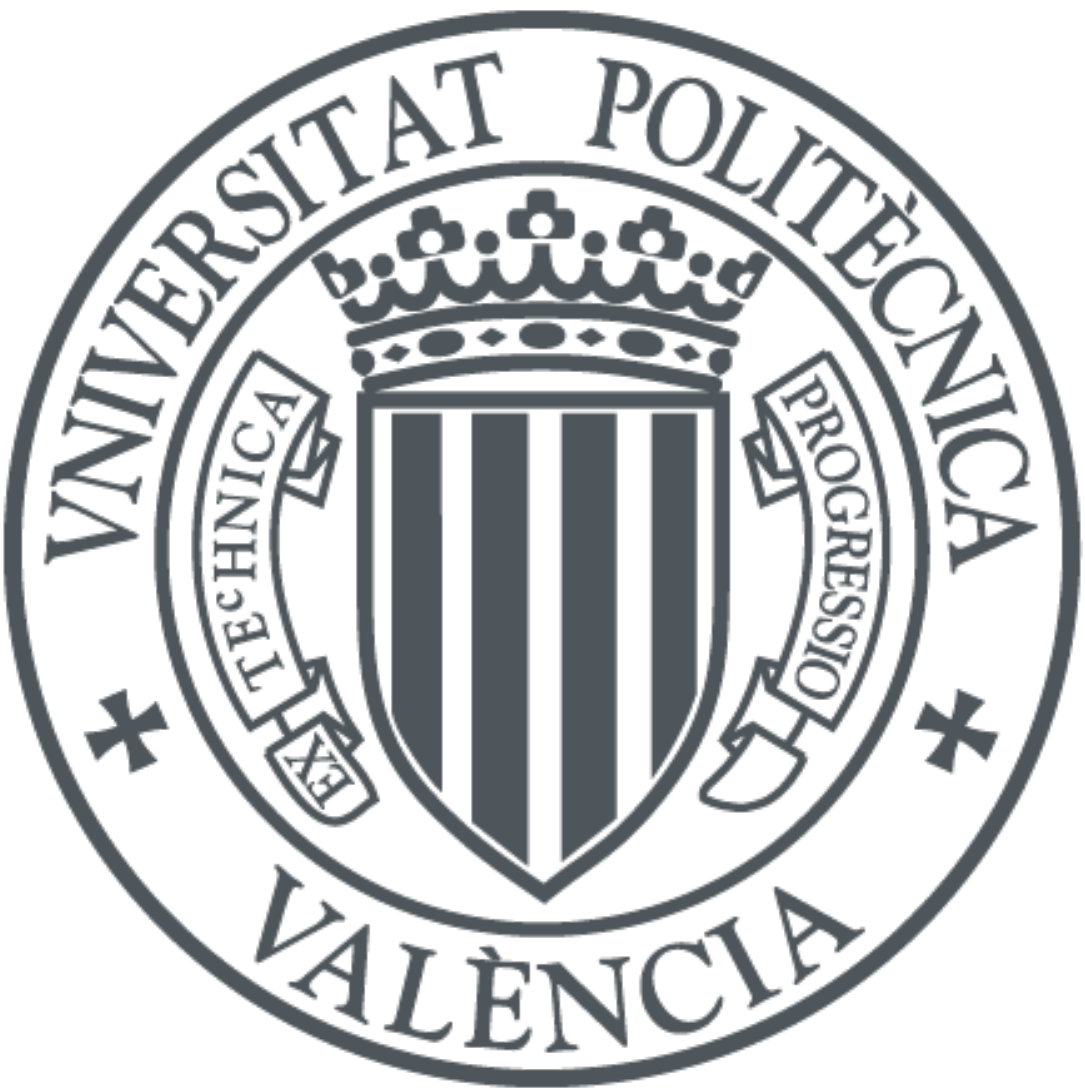

The final publication is available at

http://dx.doi.org/10.1016/j.isatra.2015.09.010

Copyright Elsevier

Additional Information 


\title{
Colorimetric detection of hazardous gases using a remotely-operated capturing and processing system
}

\author{
Roberto Montes-Robles ${ }^{a}$, María Esperanza Moragues $^{\mathrm{a}, \mathrm{b}}$, José-Luis \\ Vivancos $^{\mathrm{a}, \mathrm{b}}$, Javier Ibáñez ${ }^{\mathrm{a}}$, Rubén Fraile ${ }^{\mathrm{c}, *}$, Ramón Martínez-Máñez $^{\mathrm{a}, \mathrm{b}}$, \\ Eduardo García-Breijo ${ }^{a}$ \\ ${ }^{a}$ Centro de Reconocimiento Molecular y Desarrollo Tecnológico (IDM) \\ Unidad Mixta Universidad Politécnica de Valencia - Universidad de Valencia \\ Camino de Vera s/n. 46022 Valencia, Spain. \\ ${ }^{b}$ CIBER de Bioingeniera, Biomateriales y Nanomedicina (CIBER-BBN). \\ ${ }^{c}$ Signal Theory $\&$ Communications Department \\ Escuela Técnica Superior de Ingeniería y Sistemas de Telecomunicación \\ Universidad Politécnica de Madrid - Campus Sur \\ Carretera de Valencia km \%. 28031 Madrid, Spain.
}

\begin{abstract}
This paper presents an electronic system for the automatic detection of hazardous gases. The proposed system implements colorimetric sensing algorithms, thus providing a low-cost solution to the problem of gas sensing. It is remotely operated and it performs the tasks of image capturing and processing, hence obtaining colour measurements in RGB (Red-Green-Blue) space that are subsequently sent to a remote operator via the internet. A prototype of the system has been built to test its performance. Specifically, experiments have been carried out aimed at the detection of $\mathrm{CO}, \mathrm{CO}_{2}, \mathrm{NO}, \mathrm{NO}_{2}, \mathrm{SO}_{2}$ and formaldehyde at diverse concentrations by using a chromogenic array composed by 13 active and 2 inert compounds. Statistical analyses of the results reveal a good performance of the electronic system and the feasibility of remote hazardous gas detection using colorimetric sensor arrays.
\end{abstract}

Keywords: Gas detector, Image colour analysis, Remote sensing

\section{Introduction}

The detection of hazardous gases is important for safety reasons in a variety of industrial environments [1], ranging from the aerospace sector [2] to the

\footnotetext{
*Corresponding author

Email addresses: romonrob@etsid.upv.es (Roberto Montes-Robles), mamopon1@upvnet.upv.es (María Esperanza Moragues), jvivanco@dpi.upv.es (José-Luis Vivancos), jibanyez@eln.upv.es (Javier Ibáñez), rfraile@ics.upm.es (Rubén Fraile), rmaez@qim.upv.es (Ramón Martínez-Máñez), egarciab@eln.upv.es (Eduardo García-Breijo)
} 
oil and gas industry [3]. The application of remote gas detection in such environments has benefits related not only with the reduction of risks for human operators, but also with cost savings [4]. The facts that the presence of these gases is dangerous even at low concentrations, and that some of them cannot be perceived by the human smelling system, for instance $\mathrm{CO}$, make their detection an ongoing research issue $[5,6,7]$. Several aspects have to be taken into account in the design of remote gas detection systems, including selection of sensing and transmission technologies, choice of system architecture and decision on the degree of automation. In this paper, a system aimed at the remote detection of hazardous gases is described. A prototype of it has been built and results are presented from a laboratory experiment carried out with the purpose of checking the feasibility of its application to the detection of several gases.

Regarding sensing technology, a variety of sensor types have been proposed for gas detection up to now [8], such as electrical/electronic, acoustic, optical spectrographic, and so on. Each type has its own advantages and disadvantages in terms of cost, energy consumption, selectivity, sensitivity, etc. In recent years, colorimetric sensor arrays have been introduced as a new sensing technology for the detection of diverse compounds, both in gas and liquid phases $[9,10]$. In the case of gases, applications reported in the scientific literature are mainly related to biological processes, such as the food degradation process [11, 12, 13] or metabolic diseases [13]. The use of colorimetry for such applications provides solutions at a lower cost when compared to other approaches [10], since colour measurement can be accomplished by means of widely used devices such as flatbed scanners [14] or digital cameras [11], though specifically designed devices have also been proposed [15]. The system presented here has the novelty of extending the range of applications of colorimetric sensors to the case of hazardous gases and it includes a digital camera (a webcam) as the image capturing device.

As for transmission technology, design options may be classified into two broad classes: either wireless or wired. System architecture, for its part, will depend on a key decision regarding distance between sensing devices and controllers or actuators. For instance, if sensors are separated from controllers or actuators, wireless connections are a cost-effective solution [16]. But low energy consumption will be a strict requisite for sensors [8] in that case. If sensors and controllers or actuators are not separated, connections may be wired or even sensors be integrated with controllers or actuators [3]. In this case, a communication system that allows remote operation of such controllers or actuators should be designed. With respect to other systems, the one described here has the singularity of the sensing device being independent from the data capturing device. Since the sensing device consists in an array of materials whose colour changes in the presence of gases, and the device that captures such changes is a camera, there is no need for electronic communications between sensors and capturing device. Only line of sight between them is required. The capturing device is connected to the controller by wire and remote operation of the controller is achieved via open standards.

Last, as far as automation is concerned, the design of image capturing and 
processing systems for colorimetric sensing has to face several issues. One of such issues is the need for constancy of illumination. Some solutions based on the use of computer screens as controllable lighting devices have been proposed to solve this problem $[17,18]$. Another challenging issue is the identification of sensors' positions within the captured image and the evaluation of their colours, given that sensor surfaces may not be completely flat and that the interaction between sensing material and surrounding gas may not be uniform across the whole surface. This has been solved by some researchers through manual selection of an area within the image from which a colour measurement is obtained [19]. Both issues are solved in the herein presented system by allowing the controller to manage illumination and to automatically process captured images.

Specifically, the proposed system implements colorimetric sensing algorithms on a general-purpose minicomputer [20], thus providing a low-cost solution to the problem of gas sensing. Additionally, the use of a general-purpose minicomputer makes it possible for the same system to automatically perform the functions of lighting control, image capturing, image processing and result reporting. Furthermore, the minicomputer is equipped with a network card that connects it to the internet. This allows it to be remotely operated, therefore avoiding the need for a human operator to be present in the area where gases are to be detected. A detailed description of the proposed system is included in section 2 (subsection 2.1). A laboratory experiment was carried out in order to provide a proof of concept for the system. The colorimetric sensing array used in this paper is similar to the one described in $[11,12]$. Further details on the array and the experiments are provided in subsections 2.2 and 2.3. Detection results are presented in section 3 .

\section{Materials and methods}

\subsection{Image capturing and processing system}

\subsubsection{Hardware}

The electronic system in charge of capturing images of the chromogenic array and processing them so as to detect colour changes in the sensing materials is basically composed by a low-cost minicomputer (Raspberry Pi [20]), equipped with a webcam (RaspiCam [20]) and connected to the internet. The overall system architecture is depicted in Figure 1 and pictures of the equipment are shown in Figure 2. Table 1 includes additional technical details of the minicomputer and the camera, while the specific architecture of the lighting system is illustrated by the block diagram in Figure 3 .

The minicomputer controls, via the GPIO (General Purpose Input/Output) port, a LED (Light-Emitting Diode) lighting system. The purpose of the lighting system is to illuminate the scene at the time of image capturing. The lighting system requires a $24 \mathrm{~V}$ voltage source and a current with intensity approximately equal to $1.5 \mathrm{~A}$. However, the output port of the Raspberry Pi only provides $3.3 \mathrm{~V}$ and $20 \mathrm{~mA}$. Thus, a power stage had to be designed to serve as interface between the minicomputer and the LED lighting system. The power stage includes an 


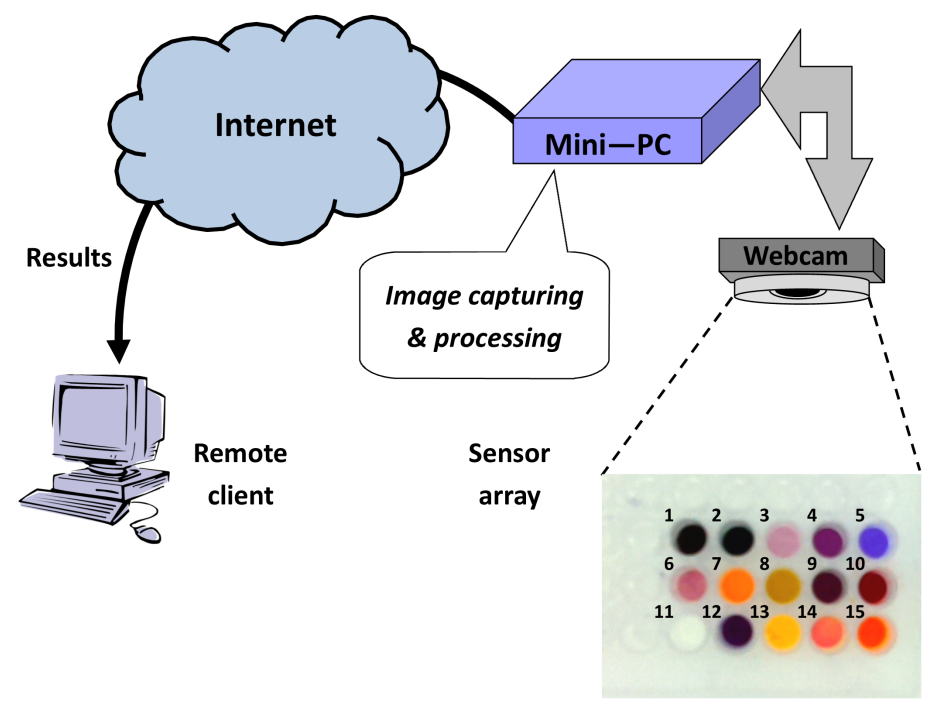

Figure 1: Overall architecture of the electronic image capturing and processing system.

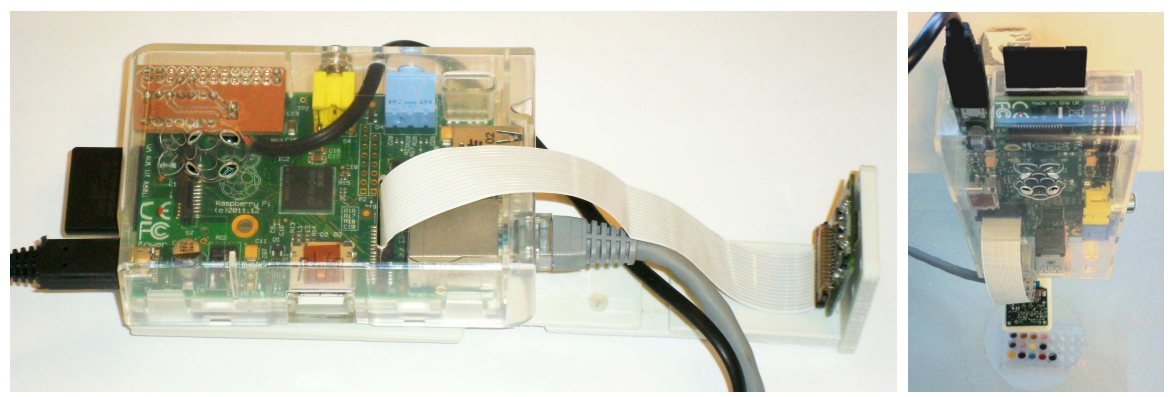

Figure 2: Picture of the equipment: detailed view (left) and top-down view with the target sensor array at the bottom (right).

\begin{tabular}{|c|c|}
\hline \multicolumn{2}{|l|}{ Minicomputer } \\
\hline Microchip (CPU): & Broadcom BCM2835 (ARM1176JZF - 700MHz) \\
\hline Graphics adapter: & VideoCore IV GPU (HD quality - 512MB RAM) \\
\hline Storage device: & SD Card \\
\hline Operating System: & Raspbian [20] (Linux-based) \\
\hline Ports: & Ethernet, GPIO, audio, HDMI \\
\hline \multicolumn{2}{|l|}{ Camera } \\
\hline $\begin{array}{r}\text { Resolution: } \\
\text { Configurable settings: }\end{array}$ & $\begin{array}{l}5 \text { Mpixel } \\
\text { ISO, brightness, contrast, saturation, etc. }\end{array}$ \\
\hline
\end{tabular}

Table 1: Technical specifications of both minicomputer and camera. 


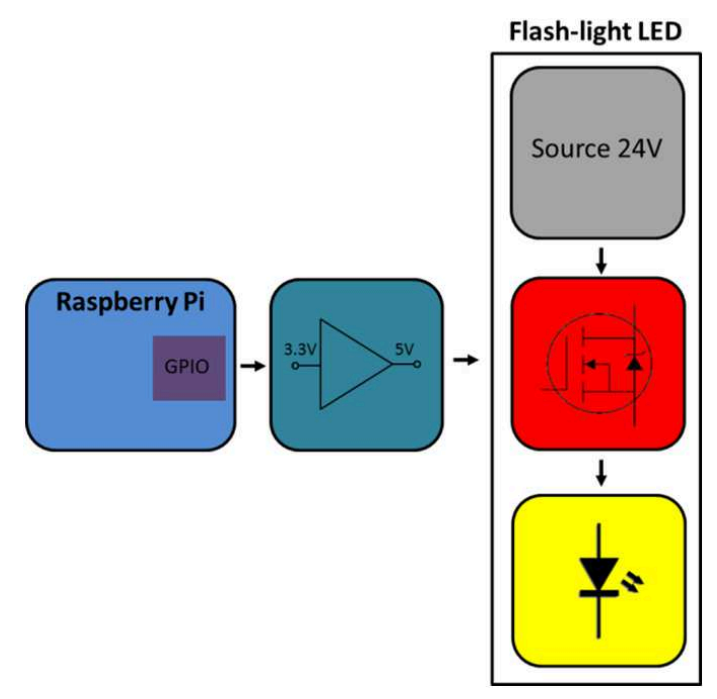

Figure 3: Block diagram of the lighting system, including the minicomputer, the power stage, the source and the LEDs.

amplifier based on the IRF640 transistor that is capable of controlling currents up to $18 \mathrm{~A}$ (red block in Figure 3 ). For the amplifier to work properly, a gatesource voltage level of at least $5 \mathrm{~V}$ is needed. Such voltage is provided by a logic level converter $(3.3 \mathrm{~V}$ to $5 \mathrm{~V})$ incorporated to the system (turquoise block in Figure 3).

\subsubsection{Image processing}

The image capturing and processing software was developed in Python 2.7 [21] and it makes use of the Open CV library [22] . Both capturing and processing routines are run on the mini-PC and results are subsequently sent via e-mail (Simple Mail Transfer Protocol [23]) to a remote server. The system can be remotely operated by making use of the standard XDMCP (X Display Manager Control Protocol) [24] and SSH (Secure SHell) [25] protocols. Yet, the joint use of a general-purpose computer and high-level programming languages makes it feasible to adapt the system to any other standard communications protocol.

A detailed description of the image processing algorithms is beyond the scope of this paper. Yet, a brief outline of the procedure for locating the probes on the captured image is provided next:

i. The colour image is first converted to a luminance matrix (see [26] for further reference on colour models and conversions). 
ii. A thresholding operation is performed to yield a binary image.

iii. After thresholding, noise reduction is achieved by a dilation operation [26].

iv. Image objects are detected by carrying out a contour detection operation on the binary image, using the algorithm implemented in Open CV.

v. For the region limited by each contour, its area and centroid coordinates are calculated.

vi. Based on the area calculations, too large and too small regions are discarded.

vii. The darkest remaining region (carbon powder, as mentioned below) is then located. This, together with the centroids positions allows for the estimation of the rotation angle of the probe matrix.

viii. Information on the rotation angle is further used in conjunction with centroids positions to locate the centre of all the probes.

\subsection{Chromogenic array}

Colorimetric sensing of gases is based on interactions between gases and sensing materials that go beyond physical adsorption. Specifically, sensing materials should be chosen whose molecules change the wavelengths of either reflected or absorbed light upon changes in their chemical environment. Such materials typically include $\mathrm{pH}$ indicators and Lewis acids, supported by some inert solids [9].

For the system presented here, sensing (chromogenic) materials were prepared by dissolving a certain amount of one out of nine different dyes in one of two types of solvent. Each solution was then added to an inorganic solid support. The dyes include eight $\mathrm{pH}$ indicators, acquired from Sigma Aldrich (St. Louis, MO, USA), and dinuclear rhodium complex, synthesized according to the procedures described in [27]. Each dye has been solved in either ethanol or dichloromethane (solvents) and added to either alumina or silica gel (supports). Both supports and solvents were purchased from Scharlab SL (Barcelona, Spain). The specific combinations of dyes, solvents and supports are provided in Table 2 for interested readers.

The chromogenic array was prepared by placing approximately $40 \mathrm{mg}$ of each of the thirteen materials enumerated in Table 2 into a well of a microplate. Two additional inert materials were also used with the purpose of providing black and white colour references for the image processing algorithms. Thus, all materials completed a $3 \times 5$ array (fifteen materials in all). The array set-up is depicted in the bottom-right end of Figure 1.

\subsection{Gases preparation}

The colour changes of the chromogenic array when surrounded by an atmosphere containing different concentrations of hazardous gases were tested. Specifically, the sensitivity of the array to $\mathrm{CO}, \mathrm{CO}_{2}, \mathrm{NO}, \mathrm{NO}_{2}, \mathrm{SO}_{2}$ and formaldehyde was assessed. Carbon monoxide was obtained from commercially available $\mathrm{CO}$ cylinders. The rest of gases were generated in situ. Gas mixtures were prepared according to the same procedures as in [15]. In brief, gas mixtures were obtained at $25^{\circ} \mathrm{C}$ by a computer-driven gas mixing system composed of two 


\begin{tabular}{|c|l|l|l|c|}
\hline Code & Dye & Solvent & Support & $\frac{\text { mg dye }}{\mathrm{mg} \text { solid }}$ (\%) \\
\hline 1 & - & - & Carbon & - \\
2 & Bromocresol green & Ethanol & Silica gel & 2 \\
3 & Binuclear rhodium complex & Dichloromethane & Silica gel & 7 \\
4 & Bromocresol purple & Ethanol & Alumina & 0.2 \\
5 & Bromophenol blue & Ethanol & Silica gel & 0.2 \\
6 & Thymol blue & Ethanol & Alumina & 2 \\
7 & Phenol red & Dichloromethane & Silica gel & 2 \\
8 & Bromothymol blue & Sthanol & Alica gel & 2 \\
9 & Bromocresol purple & Ethanol & Silica gel & 2 \\
10 & Methyl red & Ethanol & Silica gel & 2 \\
11 & - & - & Silica gel & 2 \\
12 & Bromophenol blue & Ethanol & Silica gel & 2 \\
13 & Bromocresol purple & Ethanol & Alumina & 2 \\
14 & m-Cresol purple & Ethanol & Silica gel & 2 \\
15 & m-Cresol purple & Ethanol & & \\
\hline
\end{tabular}

Table 2: Details of utilised sensing materials. Codes correspond to the well numbering in Figure 1. Each row indicates the specific combination of dye, solvent and support placed in the corresponding well of the microplate. The last column indicates the mass ratio between solved dye and support.

\begin{tabular}{r|l} 
Gas & Concentrations (ppm) \\
\hline $\mathrm{CO}$ & $0,5,50,100$ \\
$\mathrm{CO}_{2}$ & $0,5000,15000$ \\
$\mathrm{NO}$ & $0,1,10,25$ \\
$\mathrm{NO}_{2}$ & $0,1,40,100$ \\
$\mathrm{SO}_{2}$ & $0,1,10,20,100$ \\
Formaldehyde & $0,1,10,20,100$
\end{tabular}

Table 3: Gas concentrations for the first experiment.

mass flow controllers (model F-201CV, Bronkhorst High-Tech). In addition, gas concentrations were validated with a Testo analyzer (315_2 model 0632 0317), which had previously been calibrated and certified by the Spanish Certification Agency (ENAC).

Two experiments were carried out. For the first one, the chromogenic array was subjected to atmospheres with increasing concentrations of each gas. At each concentration, a colour image of the array was captured and processed by the electronic system. Once the maximum tested concentration was reached, the sensing materials were renewed before proceeding with the next gas. Table 3 summarises the concentrations for which photographic images were captured.

For the second experiment, a single gas $\left(\mathrm{NO}_{2}\right)$ was chosen, the same procedure as before was repeated three times and images were taken at a wider set of concentrations: $0.5,2,10,20,40,100$ and $150 \mathrm{ppm}$. 

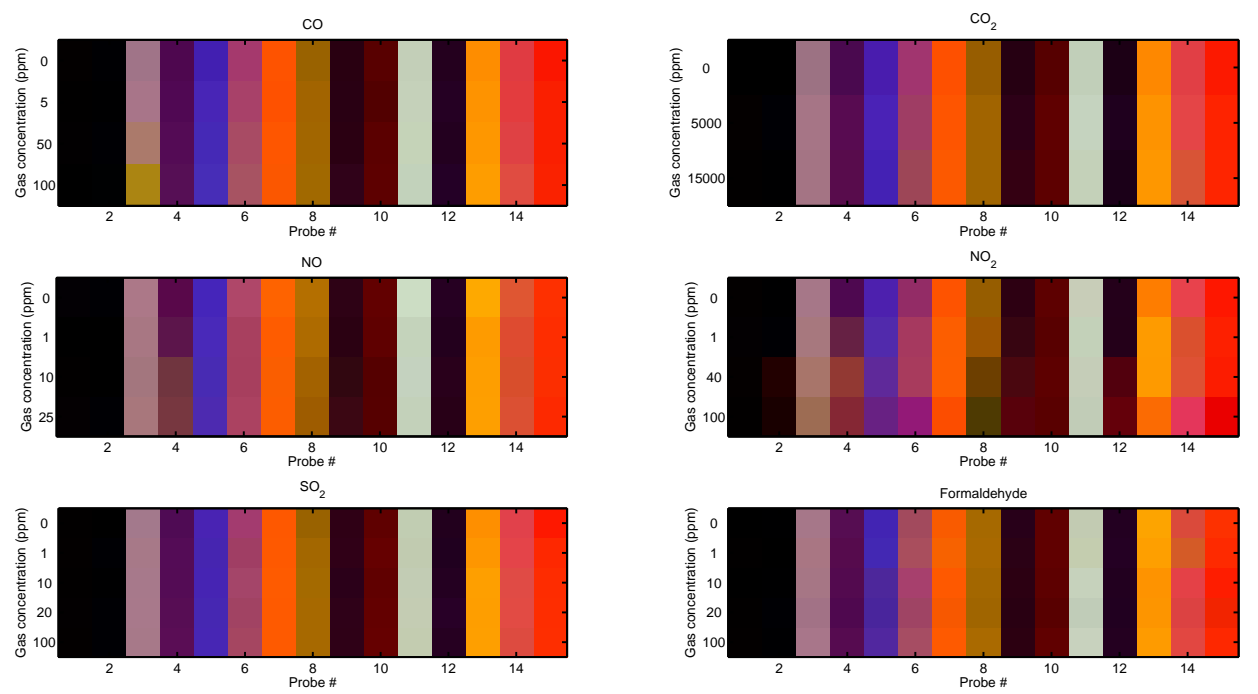

Figure 4: Colour measured at each probe and for each tested gas concentration.

\section{Results}

\subsection{Exploratory analysis}

\subsubsection{Results from the first experiment}

Figure 4 shows the colours captured by the camera for each probe and for each gas concentration tested. Probes 1 and 11 correspond to the inert compounds (carbon and silica powders respectively) used as reference black and white colours. In order to analyse colour variations, the colour distance in RGB (Red-Green-Blue) space from each measurement to the reference colour of the same probe at null gas concentration (0 ppm) has been calculated. Results have been plotted in Figure 5, where the information corresponding to the inert compounds has already been removed. From Figure 5 it can be noted that:

i. Overall, the sensing array is more sensitive to concentrations of CO, NO and $\mathrm{NO}_{2}$ than to concentrations of $\mathrm{CO}_{2}, \mathrm{SO}_{2}$ and formaldehyde.

ii. For every gas except for the formaldehyde, there exists at least a probe such that the distance of its colour to the reference colour is an increasing function of gas concentration.

iii. In general, the probes that experience the greatest colour variations are numbers $3(\mathrm{CO}), 4\left(\mathrm{NO}, \mathrm{NO}_{2}\right), 5$ (formaldehyde), $6\left(\mathrm{CO}_{2}, \mathrm{SO}_{2}\right), 8\left(\mathrm{NO}_{2}\right)$, $14\left(\mathrm{CO}_{2}\right.$, formaldehyde) and $15\left(\mathrm{SO}_{2}\right)$.

According to these observations, a principal component analysis has been carried out considering the colour distances plotted in Figure 5 corresponding to probes $3,4,5,6,8,14$ and 15 . The projection of each measurement onto the plane defined by the two first principal components is shown in Figure 6 .

From Figures 5 and 6, the following observations can be drawn: 

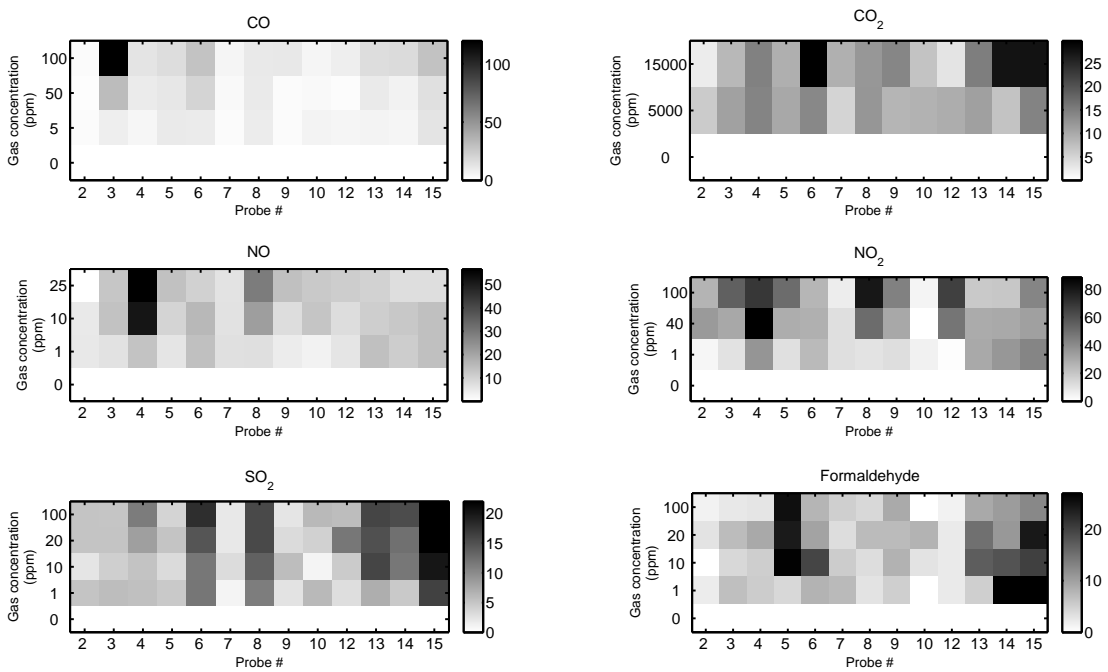

Figure 5: Colour distances in RGB space from each measurement to the colour corresponding to $0 \mathrm{ppm}$ concentration. White colour corresponds to null distance and black, to maximum distance. The bottom row at each chart corresponds to $0 \mathrm{ppm}$ concentration, so colour change is null (i.e. distance equals 0). Each chart shows how distances in colour space evolve for a different gas. Note that the maximum measurable distance in RGB colour space is equal to $255 \cdot \sqrt{3} \approx 442$.

a. The greatest variations in position happen for $\mathrm{NO}_{2}, \mathrm{NO}$ and $\mathrm{CO}$. The smallest changes occur for $\mathrm{SO}_{2}$ and $\mathrm{CO}_{2}$. The formaldehyde has an intermediate behaviour in terms of range of variation.

b. In the case of formaldehyde, the positions of measurements on the plane do not exhibit any regular relationship with gas concentration, although their associated vectors of colour distance can easily be discriminated from those corresponding to the rest of gases. This is consistent with observation ii mentioned before.

c. Variations in the concentrations of $\mathrm{NO}$ and $\mathrm{NO}_{2}$ are primarily detected by the first principal component. Its most relevant dimensions correspond to the $\mathrm{R}$ and $\mathrm{G}$ components of probes 4 and 8 and the $\mathrm{B}$ component of probes 3,4 and 5 .

d. Variations in the concentrations of $\mathrm{CO}, \mathrm{SO}_{2}$ and $\mathrm{CO}_{2}$ are primarily detected by the second principal component. Its most relevant dimensions correspond to the $\mathrm{G}$ component of probes 6,14 and 15 and the B component of probes 3 , and 5 .

e. $\mathrm{CO}_{2}$ and $\mathrm{SO}_{2}$ produce colour variations that are difficult to discriminate in terms of colour distances in RGB space.

\subsubsection{Results from the second experiment}

The second set of measurements corresponds to the three sets of measurements corresponding to $\mathrm{NO}_{2}$ and taken in different days. In each experiment, 


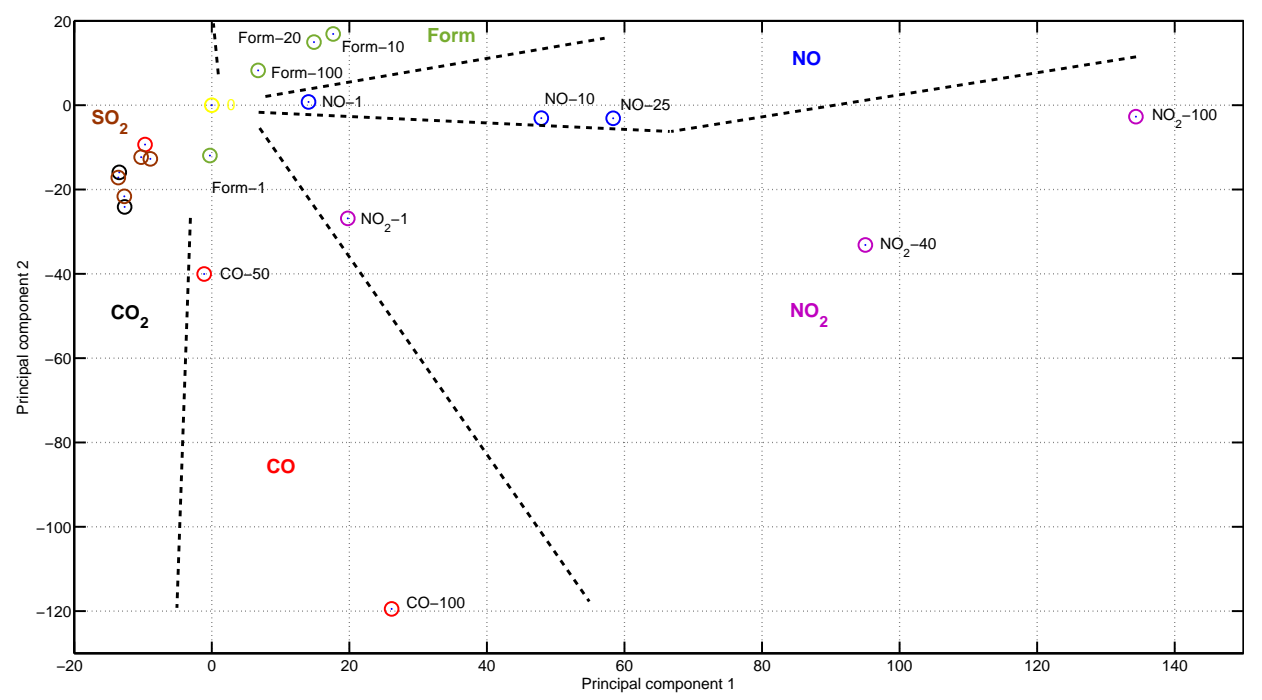

Figure 6: Projection of colour variations onto the plane defined by the two first principal components of colour distances in RGB space corresponding to probes 3, 4, 5, 6, 8, 14 and 15. A colour code has been used to identify measurements corresponding to each gas: $\mathrm{SO}_{2}$ (brown), $\mathrm{CO}_{2}$ (black), $\mathrm{CO}$ (red), $\mathrm{NO}_{2}$ (purple), $\mathrm{NO}$ (blue), formaldehyde (green). Where enough space was available, labels specifying gas and concentration have been added. Dashed lines qualitatively indicate which area within the plane correspond to each gas.

the colour changes of the probes were measured for a sequence of increasing concentrations of $\mathrm{NO}_{2}$ (from 0 to $150 \mathrm{ppm}$ ). The projection of the associated arrays of colour distances onto the plane defined by the same principal components as before (Figure 6) produces the points not marked as circles in Figure 7. It is worth noting that these points are placed in the area expected to correspond to $\mathrm{NO}_{2}$ and at a distance from the point corresponding to $0 \mathrm{ppm}$ that tends to be an increasing function of concentration.

From the previous results, it can be inferred that the array of probes exhibits a good selectivity for formaldehyde, $\mathrm{CO}, \mathrm{NO}$ and $\mathrm{NO}_{2}$ (the capability to discriminate $\mathrm{NO}_{2}$ has been checked by using a second set of measurements) and that it is also capable to detect gas concentrations to a certain extent, at least for $\mathrm{CO}, \mathrm{NO}$ and $\mathrm{NO}_{2}$ (again, the monotonic dependence of colour distances and $\mathrm{NO}_{2}$ concentration has been tested with a second set of measurements). Furthermore, the results in Figure 6 and Figure 7 indicate that a linear model with only a few variables or factors may be enough to account for the variability of the available measurements.

\subsection{Predictive analysis}

For the first set of measurements, a partial least square (PLS) model [28] of colour measurements has been built after the pre-processing of data described next. Each measurement is characterised by a vector of 39 components (measurement vector), corresponding to the $\mathrm{R}, \mathrm{G}$ and $\mathrm{B}$ components of the 13 re- 


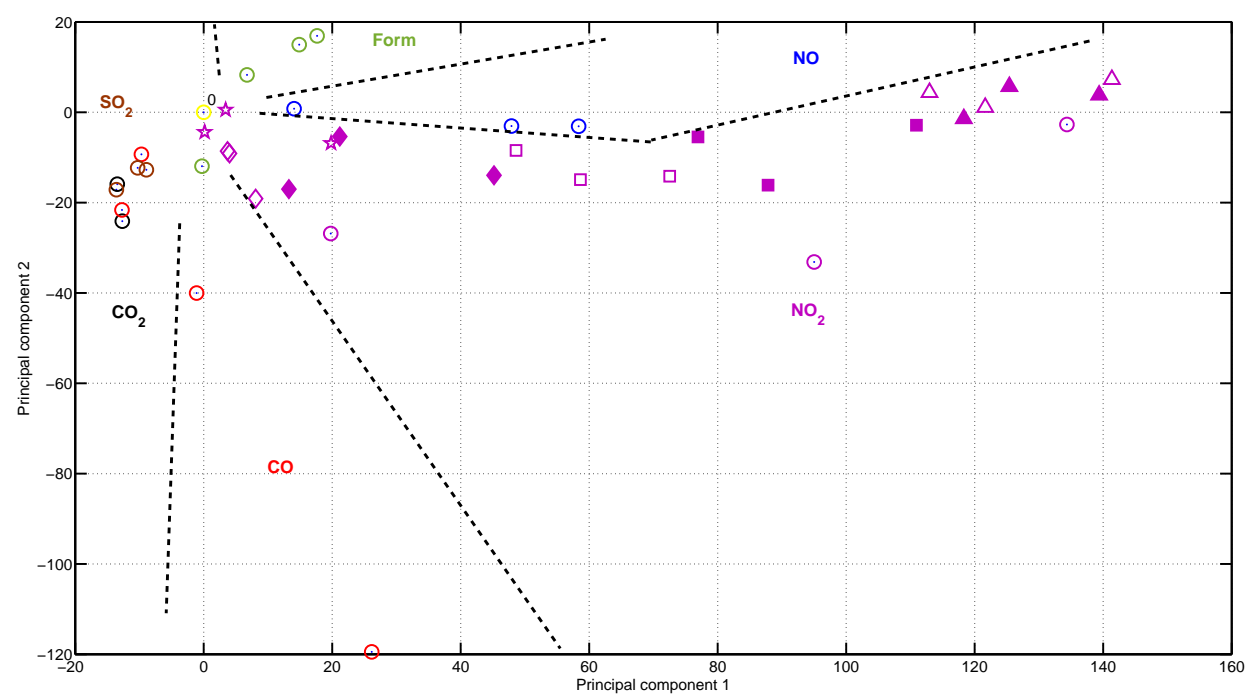

Figure 7: Projection of the second set of measurements onto the same plane as in Figure 6. For clarity, the colour code has been kept the same. Pentagram markers correspond to 0.5 ppm concentration, empty diamonds correspond to $2 \mathrm{ppm}$, full diamonds to $10 \mathrm{ppm}$, empty squares to $20 \mathrm{ppm}$, full squares to $40 \mathrm{ppm}$, empty triangles to $100 \mathrm{ppm}$ and full triangles to $150 \mathrm{ppm}$.

active probes, i.e. not considering the inert compounds. From each vector, the $\mathrm{R}, \mathrm{G}$ and $\mathrm{B}$ values corresponding to the measurement at $0 \mathrm{ppm}$ concentration have been subtracted from the corresponding components. This is necessary to achieve linearity of the system, since a null input vector (measurement vector) is to produce a null output (0 ppm). Taking into account the observation mentioned above that gas concentration tends to behave as a monotonic function of colour distance, the dimensionality of the measurement space has been reduced from 39 to 13 by computing the colour distance of each measurement to its corresponding 0-ppm point. Recall that these are the magnitudes represented in Figure 5.

Regarding gas concentrations, they have very different ranges, depending on the gas: for $\mathrm{CO}_{2}$ concentrations reach 15,000 ppm while for $\mathrm{NO}$ the maximum is $25 \mathrm{ppm}$. This would suggest a logarithmic transformation in the data, but the presence of null values $(0 \mathrm{ppm})$ makes a root more appropriate [28]. Specifically, the square root produces good results in this case. After that, each component of the measurement vector has been normalised by its standard deviation and the same normalisation has been applied to gas concentrations.

Figure 8 depicts the dependence of the fraction of explained variance of the measurements with the number of latent variables or factors in the PLS model. The breakpoint that happens for 6 latent variables is conjectured to be a threshold beyond which the model becomes over-fitted to data. For this reason, 6 has been chosen as the number of latent variables for the results reported next. 


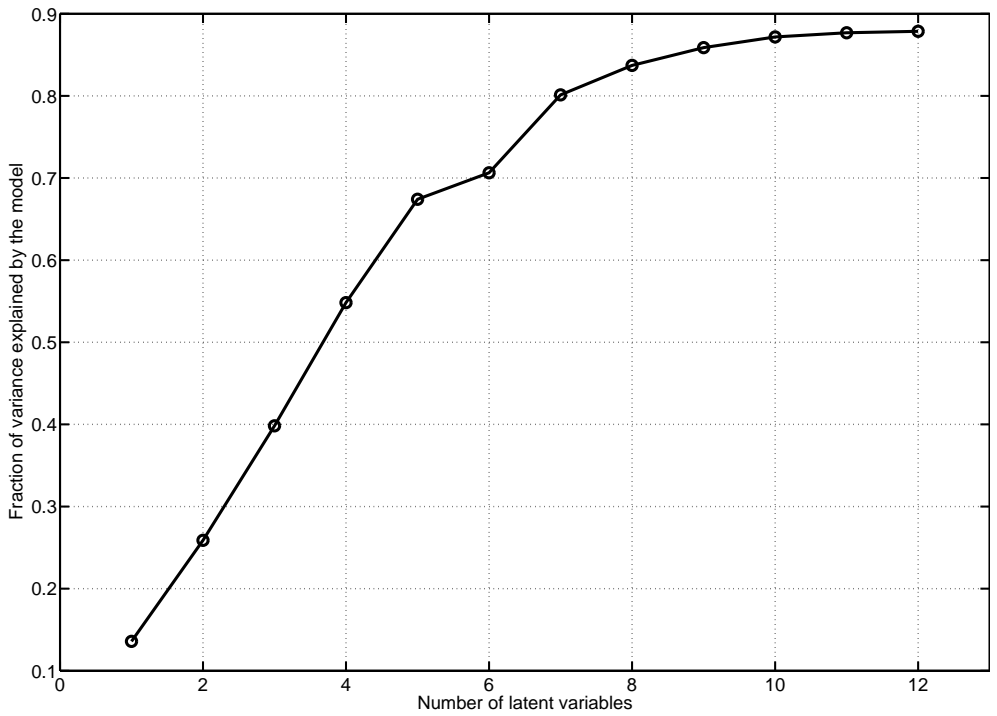

Figure 8: Fraction of variance explained by the model as a function of the number of latent variables (or factors) in the PLS model.

The PLS model built for the first set of measurements has been subsequently used to predict gas concentrations using the colour measurement vectors corresponding to the second set of measurements. The obtained results are summarised in Figures 9 and 10. For all gases, except for $\mathrm{CO}_{2}$ and $\mathrm{NO}_{2}$, the predicted concentrations are below $12 \mathrm{ppm}$ in all cases. For $\mathrm{CO}_{2}$, the maximum predicted concentration is around $800 \mathrm{ppm}$. However, since the PLS model has been built using measurements of $\mathrm{CO}_{2}$ concentration as high as 15,000 ppm, the predicted value is around $5.3 \%$ of the maximum value used for building the model and, consequently, it can also be considered as low. Thus, only predicted concentrations for $\mathrm{NO}_{2}$ reach significant values. Such results are consistent with the selectivity of the system reasoned before.

When comparing the predicted concentrations of absent gases (i.e. NO, CO, $\mathrm{CO}_{2}, \mathrm{SO}_{2}$ and formaldehyde) with some recommended limits for their air concentrations (as shown in Table 4), it can be observed that the predictions for $\mathrm{NO}, \mathrm{CO}$ and $\mathrm{CO}_{2}$ are below their corresponding thresholds. This implies that the system does not produce false alarms for these cases. On the contrary, as indicated in subsection 3.1.1, the sensing array exhibits poor selectivity with respect to $\mathrm{SO}_{2}$ and a non-regular behaviour with respect to formaldehyde concentrations. Such facts are related to the false alarms that would be produced in these two cases.

As for the results corresponding to $\mathrm{NO}_{2}$, predicted concentrations for all cases are around the measured concentrations (i.e. in the vicinity of the straight line with unit slope in the graph of Figure 10). While predictions corresponding to the same measured values exhibit a certain variability, such variability 

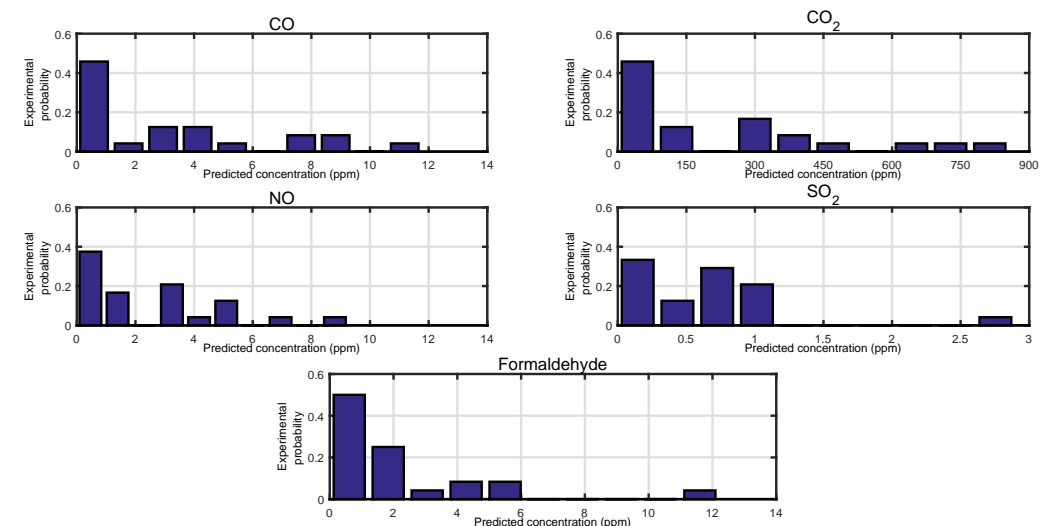

Figure 9: Histograms of predicted concentrations for all gases except for $\mathrm{NO}_{2}$; predictions correspond to the second set of measurements.

\begin{tabular}{r|l} 
Gas & Recommended limit concentrations \\
\hline $\mathrm{CO}$ & $90 \mathrm{ppm}($ during 15 minutes $)[29]$ \\
$\mathrm{CO} 2$ & $5,000 \mathrm{ppm}(8 \mathrm{~h})[30]$ \\
$\mathrm{NO}$ & $25 \mathrm{ppm}[31]$ \\
$\mathrm{NO}_{2}$ & $0.11 \mathrm{ppm}(1 \mathrm{~h})[29]$ \\
$\mathrm{SO}_{2}$ & $0.175 \mathrm{ppm}(10 \mathrm{~min})[29]$ \\
Formaldehyde & $0.33-2 \mathrm{ppm}$ (short time) $[32]$
\end{tabular}

Table 4: Some recommended limits for air concentrations of the tested hazardous gases.

becomes lower as the measured concentration grows. It should be highlighted that predicted concentrations have a monotonic behaviour with respect to measured concentrations, which indicates that the system is capable to be used to estimate concentrations of $\mathrm{NO}_{2}$.

It should also be considered that the PLS model has been built using only 4 different levels of $\mathrm{NO}_{2}$ concentrations and that it has been tested using 24 additional measurements corresponding to 8 distinct concentrations. The number of data used for training the model and their specific values of gas concentrations may explain why the prediction errors are much higher at concentrations between 0 and $10 \mathrm{ppm}$, the interval where the recommended threshold lies (Table $4)$.

\section{Conclusions}

An electronic system for the remote detection of hazardous gases has been presented in this paper. This system has the novelty of using colorimetric-based sensing. Such sensing technology has the advantage over other approaches of 


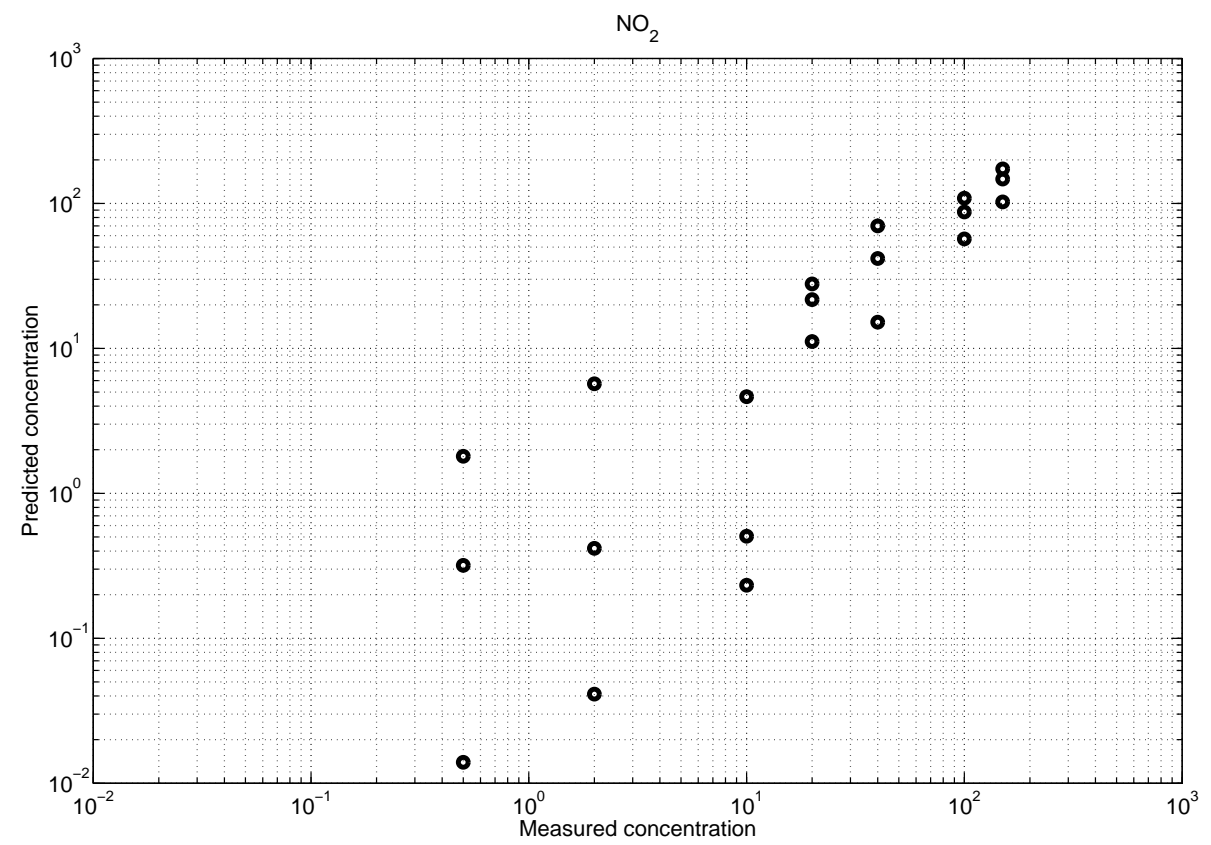

Figure 10: Predicted gas concentrations vs. measured values for $\mathrm{NO}_{2}$, corresponding to the second set of measurements.

providing a low-cost solution to gas sensing. Additionally, the use of colorimetric sensing allows human operators to assess the presence of gases by evaluating colour changes with the naked eye. Thus the same sensing technology can be used for simultaneous automatic and "manual" sensing. With respect to systems also based on colorimetric sensing and previously reported in the scientific literature, the on presented here involves a higher degree of automation. This has been achieved by incorporating image processing algorithms into the controller in charge of capturing colour information.

In order to assess the feasibility of the system, a prototype has been built using a general-purpose minicomputer equipped with a webcam and connected to the internet. System software and communications have been developed using high-level programming languages and open communication protocol standards. The fact that such a system can be designed using general-purpose hardware and programming and communication tools implies on the one hand that a complex dedicated hardware is not needed (only camera, lighting system and sensing array), so other simultaneous controlling tasks my be possibly assigned to the general purpose equipment. On the other hand, the feasibility of such approach implies that the proposed system can be integrated with controlling systems previously deployed in the plant were gas presence is to be detected.

The performance of the prototype for the remote detection of some hazardous gases has been tested. The obtained results indicate that, even using 
a sensing array that has not been specifically designed for this purpose, the system works fairly well for the detection of $\mathrm{CO}, \mathrm{NO}, \mathrm{NO}_{2}$ and formaldehyde. Additionally, good prediction results for the concentration of $\mathrm{NO}_{2}$ have been obtained. Although refinements in the design of the sensing array and the training of the prediction model are necessary to improve system performance in the neighbourhood of currently recommended limit air concentrations for the tested hazardous gases, the implemented experiments and their results provide a proof of concept for the feasibility of low-cost remote sensing of hazardous gases based on colorimetry.

\section{Acknowledgements}

This research has been carried out in the framework of project grant numbers MAT2012-38429-C04-01 and MAT2012-38429-C04-04, financed by the Spanish Ministry for Economy and Competitiveness. The authors also acknowledge the support of the Generalitat Valenciana through project grant number PROMETEOII/2014/047.

\section{References}

[1] M. H. Hammond, K. J. Johnson, S. L. Rose-Pehrsson, J. Ziegler, H. Walker, K. Caudy, D. Gary, D. Tillett, A novel chemical detector using cermet sensors and pattern recognition methods for toxic industrial chemicals, Sensors and Actuators B: Chemical 116 (1) (2006) 135-144.

[2] G. Hunter, P. Neudeck, L. Y. Chen, D. Makel, C. Liu, Q. Wu, D. Knight, A hazardous gas detection system for aerospace and commercial applications, in: AIAA/ASME/SAE/ASEE Joint Propulsion Conference and Exhibit, Cleveland, USA, 1998, pp. 1-10.

[3] G. Bonow, A. Kroll, Gas leak localization in industrial environments using a TDLAS-based remote gas sensor and autonomous mobile robot with the Tri-Max method, in: IEEE International Conference on Robotics and Automation, ICRA 2013, Karlsruhe, Germany, 2013, pp. 987-992.

[4] D. A. Anisi, C. Skourup, A. Petrochemicals, A step-wise approach to oil and gas robotics, in: IFAC Workshop on Automatic Control in Offshore Oil and Gas Production, Trondheim, Norway, 2012.

[5] T. Ahonen, R. Virrankoski, M. Elmusrati, Greenhouse monitoring with wireless sensor network, in: IEEE/ASME International Conference on Mechatronic and Embedded Systems and Applications, MESA 2008, Beijing, China, 2008, pp. 403-408.

[6] K. Wetchakun, T. Samerjai, N. Tamaekong, C. Liewhiran, C. Siriwong, V. Kruefu, A. Wisitsoraat, A. Tuantranont, S. Phanichphant, Semiconducting metal oxides as sensors for environmentally hazardous gases, Sensors and Actuators B: Chemical 160 (1) (2011) 580-591. 
[7] J. Brunet, L. Spinelle, A. Pauly, K. Dubois, M .and Guerin, M. Bouvet, C. Varenne, B. Lauron, A. Hamwi, All-organic device with integrated chemical filter dedicated to the selective measurement of no2 in air, Organic Electronics 11 (7) (2010) 1223-1229.

[8] X. Liu, S. Cheng, H. Liu, S. Hu, D. Zhang, H. Ning, A survey on gas sensing technology, Sensors 12 (7) (2012) 9635-9665.

[9] K. S. Suslick, N. A. Rakow, A. Sen, Colorimetric sensor arrays for molecular recognition, Tetrahedron 60 (49) (2004) 11133-11138.

[10] J. R. Askim, M. Mahmoudi, K. S. Suslick, Optical sensor arrays for chemical sensing: the optoelectronic nose, Chemical Society Reviews 42 (22) (2013) 8649-8682.

[11] Y. Salinas, J. V. Ros-Lis, J. L. Vivancos, R. Martínez-Máñez, M. D. Marcos, S. Aucejo, N. Herranz, I. Lorente, Monitoring of chicken meat freshness by means of a colorimetric sensor array, Analyst 137 (16) (2012) 3635-3643.

[12] P. Zaragozá, A. Fuentes, I. Fernández-Segovia, J. L. Vivancos, A. Rizo, J. V. Ros-Lis, J. M. Barat, R. Martínez-Máñez, Evaluation of sea bream (Sparus aurata) shelf life using an optoelectronic nose, Food chemistry 138 (2) (2013) 1374-1380.

[13] Z. Tang, J. Yang, J. Yu, B. Cui, A colorimetric sensor for qualitative discrimination and quantitative detection of volatile amines, Sensors 10 (7) (2010) 6463-6476.

[14] L. Feng, C. J. Musto, K. S. Suslick, A simple and highly sensitive colorimetric detection method for gaseous formaldehyde, Journal of the American Chemical Society 132 (12) (2010) 4046-4047.

[15] M. E. Moragues, R. Montes-Robles, J. V. Ros-Lis, M. Alcañiz, J. Ibáñez, T. Pardo, R. Martínez-Máñez, An optoelectronic sensing device for CO detection in air based on a binuclear rhodium complex, Sensors and Actuators B: Chemical 191 (2014) 257-263.

[16] P. C. Jain, R. Kushwaha, Wireless gas sensor network for detection and monitoring of harmful gases in utility areas and industries, in: International Conference on Sensing Technology, ICST 2012, Kolkata, India, 2012, pp. 642-646.

[17] D. Filippini, S. P. S. Svensson, I. Lundström, Computer screen as a programmable light source for visible absorption characterization of (bio)chemical assays, Chemical Communications (2003) 240-241.

[18] D. Filippini, A. Alimelli, C. Di Natale, R. Paolesse, A. D'Amico, I. Lundström, Chemical sensing with familiar devices, Angewandte Chemie International Edition 45 (23) (2006) 3800-3803. 
[19] S. Lakkis, R. Younes, M. Ghandour, Y. Alayli, New optical gas sensor for gas concentration measurement using digital image processing, Sensors and Actuators B: Chemical 207, Part A (2015) 321-329.

[20] Raspberry Pi Foundation, Raspberry pi, (viewed: 21 April 2014).

URL http://www.raspberrypi.org

[21] The Python Software Foundation, Welcome to Python.org, (viewed: 06 October 2014).

URL https://www.python.org

[22] Itseez, Open CV, (viewed: 06 October 2014).

URL http://opencv.org

[23] Network Working Group, Simple mail transfer protocol, RFC 5321, Internet Engineering Task Force (2008).

URL https://tools.ietf .org/html/rfc5321

[24] X Consortium, Laboratory for Computer Science, X display manager control protocol, X.Org Standard v1.1, Massachusetts Institute of Technology (2004).

URL http://www.x.org/releases/X11R7.6/doc/libXdmcp/xdmcp.html

[25] Network Working Group, The secure shell (SSH) transport layer protocol, RFC 4253, Internet Engineering Task Force (2006).

URL https://tools.ietf.org/html/rfc4253

[26] R. C. González, R. E. Woods, S. L. Eddins, Digital image processing using MATLAB, Pearson Prentice-Hall, Upper Saddle River, NJ, 2004.

[27] M. E. Moragues, J. Esteban, J. V. Ros-Lis, R. Marínez-Máñez, M. D. Marcos, M. Martínez, J. Soto, F. Sancenón, Sensitive and selective chromogenic sensing of carbon monoxide via reversible axial $\mathrm{CO}$ coordination in binuclear rhodium complexes, Journal of the American Chemical Society 133 (39) (2011) 15762-15772.

[28] S. Wold, M. Sjöström, L. Eriksson, PLS-regression: a basic tool of chemometrics, Chemometrics and Intelligent Laboratory Systems 58 (2) (2001) 109-130.

[29] World Health Organization, Air quality guidelines for europe, WHO regional publications. European series. 91 (2000).

URL http://www.euro.who.int/en/publications/abstracts/

air-quality-guidelines-for-europe

[30] Occupational hazards of carbon dioxide exposure, Journal of Chemical Health and Safety 16 (2) (2009) 18-22.

[31] W. J. Hunter, G. Aresini, R. Haigh, P. Papadopoulos, W. Von der Hude, Occupational exposure limits for chemicals in the European Union, Occupational and Environmental Medicine 54 (4) (1997) 217-222. 
[32] K. H. Kim, S. A. Jahan, J. T. Lee, Exposure to formaldehyde and its potential human health hazards, Journal of Environmental Science and Health, Part C 29 (4) (2011) 277-299. 